\title{
The Impact of Double Fixation with Titanium Tack and N-Butyl Cyanoacrylate Glue (NBCG) Mesh Fixation versus NBCG Fixation Only in Totally Extra-Peritoneal Hernioplasty with 3-Dimensional Configured Polyester Mesh: A Comparative Study
}

Kejin Chen, M.S. ${ }^{1}$, Jianwen Liu, M.S. ${ }^{1}$ Xuefei Yang, M.D. ${ }^{1}$, Joe King Man Fan, MBBS MS (HKU) FRCSEd ${ }^{1,2}$

Department of Surgery, 'The University of Hong Kong-Shenzhen Hospital, Shenzhen, ${ }^{2}$ Li Ka Shing Faculty of Medicine, The University of Hong Kong, HKSAR, China

Purpose: Our aim is to compare 3-dimensional mesh fixation using titanium tacks combine with n-butyl cyanoacrylate glue (NBCG) (COMBINE group) versus NBCG only (NBCG group) in totally extraperitoneal inguinal hernioplasty (TEP).

Methods: This is a retrospectively study of patients diagnosed with unilateral inguinal hernia and underwent TEP with 3-dimensional configured polyester mesh fixation using titanium tacks combine NBCG or NBCG only at the University of Hong Kong-Shenzhen Hospital with data prospectively collected. Operative details and outcomes were compared including: operating time, size of defect, total hospital cost, post-operative pain scores and recurrence.

Results: From 08.2013 to 03.2016 a total of 219 patients were included. There was no significant difference between COMBINE group and NBCG group in mean age (52.5 years versus 48.2 years), mean size of defects $(2.4 \mathrm{~cm}$ versus $2.6 \mathrm{~cm}$ ), and operating time (121 mins versus 111 mins). There were significant differences between COMBINE group and NBCG group in total hospital cost (3035 USD versus 2022 USD), post-operative pain score on day 2 to day 4 (VAS: 1.4 versus $1.0,1.0$ versus $0.4,0.5$ versus 0.2). There was one recurrence in COMBINE group ( $p=0.276)$ with overall recurrence of $0.46 \%$.

Conclusion: Patients with inguinal hernia underwent TEP with 3-dimensional configured polyester mesh with NBCG fixation only having comparative surgical outcome to COMBINE group. A recurrence of $0.46 \%$ can be achieved with this combination. Tacks fixation may cause more postoperative pain and increase hospital cost. Use of $\mathrm{N}$-butyl cyanoacrylate glue in TEP is safe and effective in our clinical series.

Keywords: Inguinal hernia, Mesh fixation, Titanium tacks, N-Butyl Cyanoacrylate Glue, Laparoscopic

This is an Open Access article distributed under the terms of the Creative Commons Attribution Non-Commercial License (http:// creativecommons.org/licenses/by-nc/4.0/) which permits unrestricted non-commercial use, distribution, and reproduction in any medium, provided the original work is properly cited.
Received December 29, 2017

Revised July 11, 2018

Accepted July 30, 2018

Corresponding author

Joe King Man Fan

Department of Surgery, The

University of Hong Kong, Pokfulam

Road, Hong Kong

Tel: +852-22554763

Fax: $+852-28728425$

E-mail: drjoefan@hku.hk ORCID:

http://orcid.org/0000-0001-6604-2295
Copyright $\odot 2018$ The Journal of Minimally Invasive Surgery. All rights reserved.

\section{INTRODUCTION}

Inguinal hernioplasty is one of the most commonly performed procedures worldwide. ${ }^{1,2}$ Laparoscopic hernioplasty offers benefits in terms of early recovery and lower postoperative complications, ${ }^{3,4}$ minimize the trauma for bilateral inguinal hernia repair, and are excellent for recurrent hernia management following previous open repair. ${ }^{5}$ Laparoscopic 
hernioplasty, therefore, is becoming the gold standard for hernia repair in these circumstances. ${ }^{4,6}$

Fixation of prosthetic mesh with titanium tacks in laparoscopic hernioplasty helps to reduce recurrence. ${ }^{1,7}$ However it is reported that it may associate with increase in post-operative pain and discomfort. Therefore numerous studies have been focused on the use of alternative approaches for mesh fixation, such as use of suture or fibrin sealant glue fixation. We therefore try to explore the most optimal and most economical surgical approach for mesh fixation after totally extra-peritoneal hernioplasty and yet to achieve the best surgical outcome. The newer generation of polyester mesh with a 3-dimension configuration will remain its original position after extraperitoneal space is deflated, however, addition fixation method may be necessary in order to further minimize the recurrence rate. We fix the mesh with titanium tacks over medial and superior borders and use n-butyl cyanoacrylate glue to seal the inferior edge where closed to deep inguinal ring and triangle of Doom. Double fixation is unique and never been described before, theoretically it further reduces the chance of medial and indirect recurrence but is it cost effective for such an innovative idea? Our study aim is to compare 3-dimensional mesh fixation using titanium tacks combine with NBCG (COMBINE group) versus NBCG only (NBCG group) in totally extra-peritoneal inguinal hernioplasty.

\section{MATERIALS AND METHODS}

This is a prospectively data collected, retrospectively re- viewed study of patients diagnosed with unilateral inguinal hernia and underwent total extra-peritoneal hernioplasty with 3-dimensional configured polyester mesh (Parietex ${ }^{\mathrm{TM}} \mathrm{Hy}^{-}$ drophilic Anatomical Mesh, Covidien-Medtronic ${ }^{\circledR}$, US) at the University of Hong Kong - Shenzhen Hospital, Shenzhen, China.

\section{Inclusion criteria}

All patient with first occurrence of unilateral inguinal hernia underwent laparoscopic totally extra-peritoneal hernioplasty (TEP), using 3-dimensional configured polyester mesh, and fixation with n-butyl cyanoacrylate glue alone or together with titanium tacks fixation are included in our study (Fig. 1).

\section{Exclusion criteria}

Patients with recurrent inguinal hernia, bilateral inguinal hernia, open procedure and laparoscopic convert to open hernioplasty, trans-abdominal pre-peritoneal prosthetic (TAPP), use other non-3-dimensional mesh, no use n-butyl cyanoacrylate glue for fixation, fixation with titanium tacks only, no documentation on fixation method were excluded from the study and analysis.

\section{Ethics}

Verbal consents were obtained from patients for data collection pre and post-operatively for research and study purposed

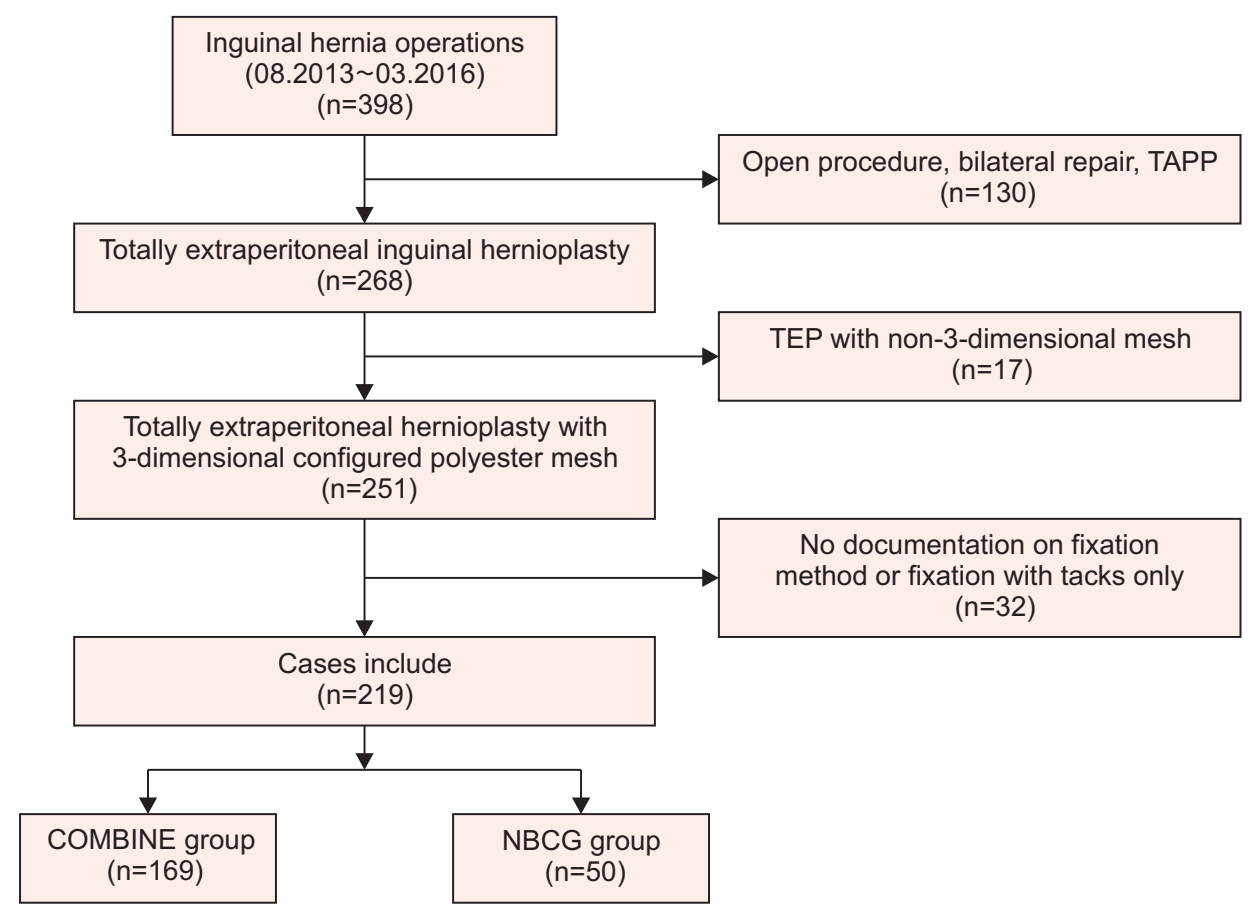

Fig. 1. Design of study. 
with identities kept confidential.

\section{Surgical outcomes}

The patients were divided into two groups based on fixation method: COMBINE group, wherein the mesh was fixed using titanium tacks over superior and medial aspects of mesh combines NBCG apply to inferior edge where closed to deep inguinal ring and triangle of Doom. NBCG group, wherein the mesh was fixed with NBCG only over superior, medial and inferior aspects. Operative details and outcome are compared including: operating time, size of defect, total hospital cost, post-operative pain scores and recurrence.

\section{Operative details}

The technique of surgery is described as follows: The patient under general anesthesia and placed supine position with both arms adducted. Antibiotics is not routinely given unless for high-risk group such as patients with diabetes mellitus. The laparoscopic unit is placed at the caudal side of the patient. A sub-umbilical transverse skin incision is made slightly towards the operative side after skin prepared with chlorohexdine solution as disinfectant. Anterior rectus sheath incision at operative side just near midline and $10 \mathrm{~mm}$ port is placed. A $30^{\circ}$ optical laparoscope inserted for visualization and preperitoneal dissection. Two $5 \mathrm{~mm}$ ports inserted at midline below $10 \mathrm{~mm}$ port under direct laparoscopic view. After trocars are inserted, the patient is placed in the Trendelenburg position. Carbon dioxide is insufflated at a pressure of $12 \mathrm{mmHg}$.

Extra-peritoneal space is then created by blunt dissection with laparoscope. Any of the direct sac is then reduced at this stage. The space of Borgros is entered and opened from lateral edge of inferior epigastric vessels. Deep transversalis fascia and acurate ligament is divided and pre-peritoneal space is

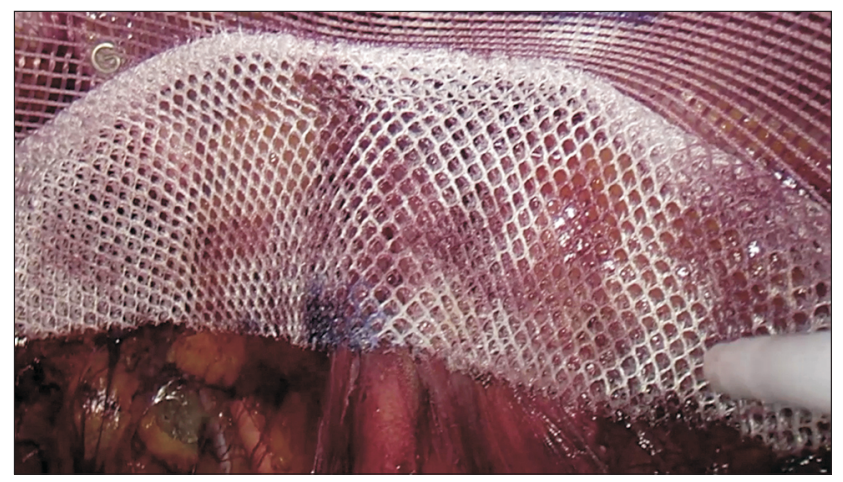

Fig. 2. Using combined approach with titanium tack and n-butyl cyanoacrylate glue for mesh fixation. created up to level of umbilicus over lateral side. Testicular vessels and vas deference are skeletonized and indirect sac is reduced or transected, with peritoneal edge is retracted at least $4 \mathrm{~cm}$ from deep inguinal ring. A 3-dimensional configured polyester mesh is put into the extra-peritoneal space with overlapping of defects at least $2 \mathrm{~cm}$. For COMBINE group patients, the mesh were fixed with a titanium tacks (ProTack ${ }^{\mathrm{TM}}$ Fixation Device, Covidien - Medtronic ${ }^{\circledR}$, US) over superior and medial aspects combine NBCG (Compont ${ }^{\mathrm{TM}}$ Medicinal Adhesive Glue, Compont ${ }^{\circledR}$, PRC) over inferior aspect of mesh near deep ring and triangle of Doom (Fig. 2). For NBCG group patients, the mesh was fixed with NBCG only over all aspects (Fig. 3).

Post-operatively, the patients were allowed to resume normal diet 4 hours after surgery. Paracetamol 1,000 mg tablets every 8 hourly for pain control in all cases.

Statistical Analysis: Results in 2 groups of patients were expressed in mean and standard deviation for continuous variables, $p<0.05$ is considered as statistically significant. The statistical analysis was carried out with licensed statistical software (SPSS ${ }^{\circledast}$ version 22.0, IBM $^{\circledR}$ Corporation, USA).

\section{Data collection and follow-up}

The patients were asked to report their pain score on a visual analogue scale (VAS) on the first 5 post-operative days with standardized self-reporting charts. Patients were assessed in out-patient clinic with standardize follow-up protocol at post-operative 1 week, 1 month and 3 months, patients were then interviewed by telephone at post-operative 1 year.

\section{RESULTS}

From 08.2013 to 03.2016 a total of 398 patients were operated for their inguinal hernia, which 219 patients diagnosed with primary, reducible, unilateral inguinal hernia and underwent

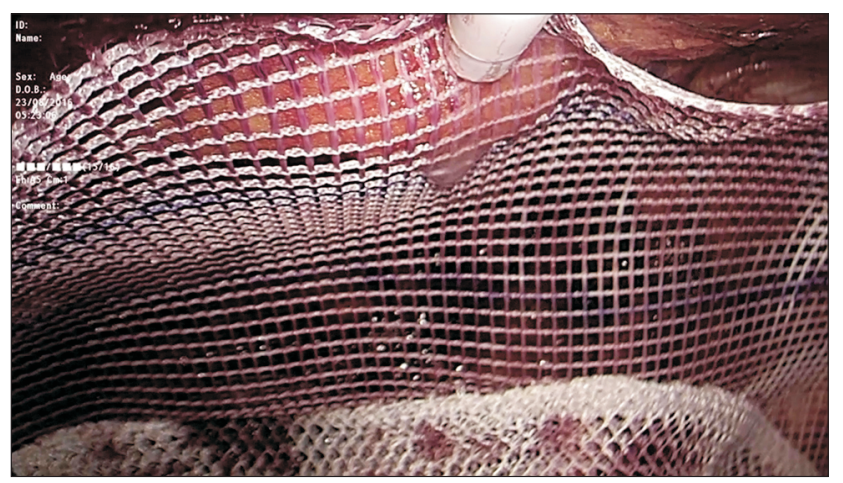

Fig. 3. Using n-butyl cyanoacrylate glue only for mesh fixation. 
Table 1. Patients demographics

\begin{tabular}{lccc}
\hline & COMBINE group & NBCG group & Significance $(p$ value) \\
\hline Total number of patients included & 169 & 50 & \\
\hline Age, years, median (range) & $52.5 \pm 16.5(18 \sim 88)$ & $48.2 \pm 16.6(18 \sim 78)$ & 0.776 \\
Mean size of defects $(\mathrm{cm})+$ - - s.d. & $2.4 \pm 0.7$ & $2.6 \pm 0.9$ & 0.466 \\
Gender, male/female & $151 / 6$ & $47 / 3$ & 0.511 \\
Inguino-scrotal hernia (percentage) & $29(17.2 \%)$ & $9(18.0 \%)$ & 0.959 \\
Co-morbidities & Hypertension: $n=21$ & Hypertension: $n=5$ & 0.648 \\
& Diabetes: $n=9$ & Diabetes: $n=2$ & 0.711 \\
& Benign prostatic hypertrophy: $n=4$ & Benign prostatic hypertrophy: $n=1$ & 0.883 \\
\hline
\end{tabular}

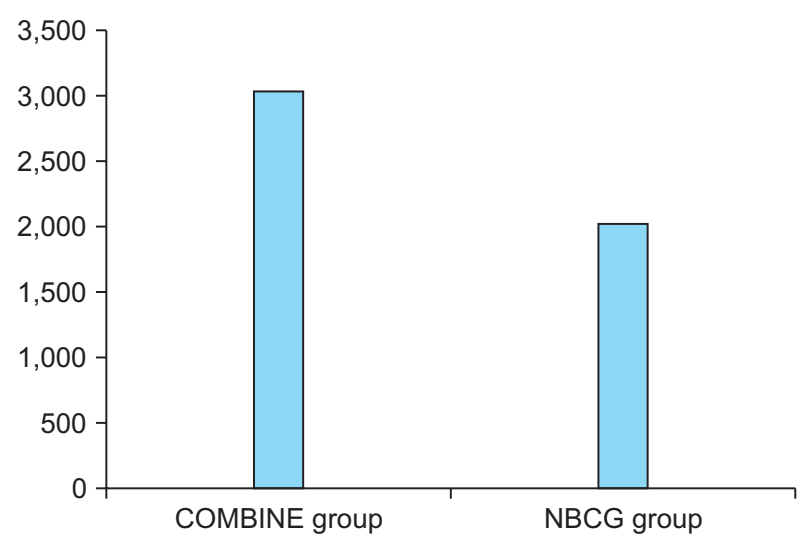

Fig. 4. Total hospital cost, USD.

TEP inguinal hernioplasty with 3-dimensional configured polyester mesh were included in our study. The age ranged from 18 to 88 years. The patient demographics are summarized in Table 1. Mean age of COMBINE group versus NBCG group is 52.5 and 48.2 years old ( $p=0.776)$. Mean size of defects was $2.4 \mathrm{~cm}$ in COMBINE group versus $2.6 \mathrm{~cm}$ in NBCG group $(p=0.466)$. There was also no significant difference in operating time (121 minutes for COMBINE group and 111 minutes for NBCK group, $p=0.331$ ). However there were significant difference in total hospital cost (USD \$3035 for COMBINE group versus USD \$2022 for NBCG group, $p=0.032$ ) (Fig. 4), and post-operative pain score on day 2 (visual analog score VAS 1.4 versus 1.0 in NBCG group, $p=0.000$ ), day 3 (VAS 1.0 versus $0.4, p=0.019$ ), and day 4 (VAS 0.5 versus $0.2, p=0.000$ ) (Fig. 5). Post-operative pain score on 1 year follow-up (visual analog score VAS 0.05 versus 0.08 in NBCG group, $p=0.118$ ), which was no significant difference between two group. There was no difference in hernia recurrence in the series with only 1 patient developed recurrence at medial aspect in COMBINE group ( $p=0.276$ ), with overall recurrence rate of $0.46 \%$ (Table 2 ).

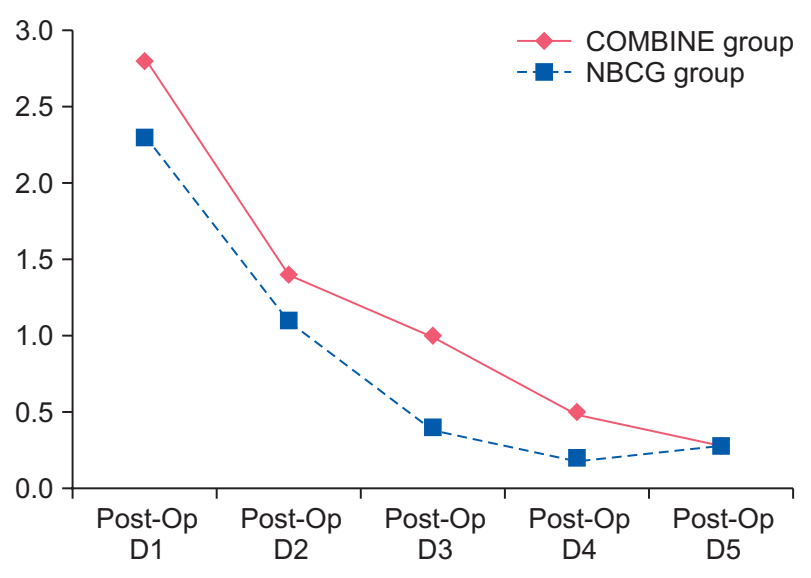

Fig. 5. Early post-operative pain score (VAS).

\section{DISCUSSION}

Although it has been shown in retrospective review, randomized study and meta-analysis that fixation of the mesh is not necessary in totally extra-peritoneal hernioplasty, ${ }^{8-12}$ but most surgeons still prefer the use of fixation adjuncts in patients with large defects or in patients with combined hernia. Most commonly used fixation method is the use of either titanium or absorbable tackers, which may have associated with increased in incidence of chronic pain and potential nerve injuries. As compared to tackers, fibrin glue fixation has been used to reduced incidence of neuralgic pain and chronic groin pain, but yet as effective as using tackers fixation. ${ }^{13-16} \mathrm{How}^{-}$ ever, cost is another concern for using human fibrin sealant for mesh fixation.

Cyanoacrylate is the generic name of a group of fast-acting adhesives material. There are three commercially available alkyl cyanoacrylates: N-butyl cyanoacrylate, $\mathrm{N}$-hexyl cyanoacrylate, $\mathrm{N}$-octyl cyanoacrylate. $\mathrm{N}$-butyl cyanoacrylate is a butyl polymer of cyanoacrylate, which undergoes an exo- 
Table 2. Operative outcomes

\begin{tabular}{|c|c|c|c|}
\hline & COMBINE group & NBCG group & $p$ value \\
\hline Operating time (mins) +/- s.d. & $121 \pm 48$ & $111 \pm 38$ & 0.331 \\
\hline Post-Op VAS* on day 1 & $2.8 \pm 1.2$ & $2.3 \pm 1.0$ & 0.056 \\
\hline Post-Op VAS* on day 2 & $1.4 \pm 1.1$ & $1.0 \pm 1.2$ & 0.000 \\
\hline Post-Op VAS* on day 3 & $1.0 \pm 1.1$ & $0.4 \pm 1.0$ & 0.019 \\
\hline Post-Op VAS* on day 4 & $0.5 \pm 0.8$ & $0.2 \pm 0.6$ & 0.000 \\
\hline Post-Op VAS* on day 5 & $0.3 \pm 0.7$ & $0.3 \pm 0.7$ & 1.000 \\
\hline Post-Op VAS* on 1 year ${ }^{*}$ & $0.08 \pm 0.27$ & $0.05 \pm 0.24$ & 0.118 \\
\hline Total hospital cost, USD & $3,035 \pm 949$ & $2,022 \pm 403$ & 0.032 \\
\hline Mean follow up, months, (range) & $21.6 \pm 7.8(13 \sim 44)$ & $23.4 \pm 7.9(12 \sim 38)$ & 0.186 \\
\hline Recurrence & 1 & 0 & 0.276 \\
\hline
\end{tabular}

Overall recurrence rate: $0.46 \%$. *Visual Analogue Scale of pain score. "Telephone follow-up.

thermic reaction in the presence of water to form a bond in about 5 seconds. The film of glue is eliminated by hydrolytic breakdown. After applying N-butyl cyanoacrylate, the mesh has to be held against the underlying tissue to achieve a secure fixation effect. With the formation of formaldehyde, n-butyl cyanoacrylate was being questioned on its safety in the clinical application in mesh fixation for hernioplasty, however it has been proven that it helps to promote mesh fixation and its safe in in-vivo study. ${ }^{17}$ In fact, cyanoacrylate glue was been used for open hernioplasty since 2003 before the era of polylactic hook self adhesive mesh, which was proven to be as effective as suture Lichtenstein hernioplasty. ${ }^{18}$ There are increasing clinical interest in the use of $n$-butyl cyanoacrylate for mesh fixation after laparoscopic hernioplasty, with the clinical advantage of potential decrease in post-operative pain and yet reduced the total cost of whole hospital stay. ${ }^{15,19-21}$

Our study shows that fixation with 3-dimensional configured polyester mesh in TEP can achieve a superior shortterm outcome in terms of recurrence despite conclusion from meta-analysis that TEP is associated with higher recurrence rate from open hernioplasty. ${ }^{22}$ Additional tack fixation has no significant impact on overall improvement in outcome of surgery but yet may cause more post operative pain and increase hospital cost. This is the first literature to compare the use of double fixation versus single fixation method in laparoscopic inguinal hernioplasty. With N-butyl cyanoacrylate glue and 3-dimensional configured mesh, we believe hernia recurrence can be further reduced if extra-peritoneal dissection is performed correctly in a standardize manner.

\section{CONCLUSION}

Patients with inguinal hernia underwent TEP with 3-dimensional configured polyester mesh with NBCG fixation only having comparative surgical outcome to COMBINE group. A recurrence of $0.46 \%$ can be achieved with this combination. Tacks fixation may cause more post-operative pain and increase hospital cost. Use of N-butyl cyanoacrylate glue in TEP is safe and effective in our clinical series.

\section{REFERENCES}

1) Bittner R, Arregui ME, Bisgaard T, et al. Guidelines for laparoscopic (TAPP) and endoscopic (TEP) treatment of inguinal hernia [International Endohernia Society (IEHS)]. Surg Endosc 2011;25: 2773-2843.

2) Lomanto D, Cheah WK, Faylona JM, et al. Inguinal hernia repair: toward Asian guidelines. Asian J Endosc Surg 2015;8:16-23.

3) Kockerling F, Stechemesser B, Hukauf M, Kuthe A, Schug-Pass C. TEP versus Lichtenstein: Which technique is better for the repair of primary unilateral inguinal hernias in men? Surg Endosc 2016; 30:3304-3313.

4) Simons MP, Aufenacker T, Bay-Nielsen M, et al. European Hernia Society guidelines on the treatment of inguinal hernia in adult patients. Hernia 2009;13:343-403.

5) Bittner R, Schwarz J. Inguinal hernia repair: current surgical techniques. Langenbecks Arch Surg 2012;397:271-282.

6) Carter J, Duh QY. Laparoscopic repair of inguinal hernias. World J Surg 2011;35:1519-1525.

7) Mayer F, Niebuhr H, Lechner M, et al. When is mesh fixation in TAPP-repair of primary inguinal hernia repair necessary? The register-based analysis of 11,230 cases. Surg Endosc 2016;30:4363- 
4371.

8) Claus CM, Rocha GM, Campos AC, et al. Prospective, randomized and controlled study of mesh displacement after laparoscopic inguinal repair: fixation versus no fixation of mesh. Surg Endosc 2016;30:1134-1140.

9) Koch CA, Greenlee SM, Larson DR, Harrington JR, Farley DR. Randomized prospective study of totally extraperitoneal inguinal hernia repair: fixation versus no fixation of mesh. Jsls 2006;10: 457-460.

10) Garg P, Rajagopal M, Varghese V, Ismail M. Laparoscopic total extraperitoneal inguinal hernia repair with nonfixation of the mesh for 1,692 hernias. Surg Endosc 2009;23:1241-1245.

11) Tam KW, Liang HH, Chai CY. Outcomes of staple fixation of mesh versus nonfixation in laparoscopic total extraperitoneal inguinal repair: a meta-analysis of randomized controlled trials. World J Surg 2010;34:3065-3074.

12) Teng YJ, Pan SM, Liu YL, et al. A meta-analysis of randomized controlled trials of fixation versus nonfixation of mesh in laparoscopic total extraperitoneal inguinal hernia repair. Surg Endosc 2011;25:2849-2858.

13) Novik B, Hagedorn S, Mork UB, Dahlin K, Skullman S, Dalenback J. Fibrin glue for securing the mesh in laparoscopic totally extraperitoneal inguinal hernia repair: a study with a 40-month prospective follow-up period. Surg Endosc 2006;20:462-467.

14) Schwab R, Willms A, Kroger A, Becker HP. Less chronic pain following mesh fixation using a fibrin sealant in TEP inguinal hernia repair. Hernia 2006;10:272-277.
15) Khaleal F, Berney C. The role of fibrin glue in decreasing chronic pain in laparoscopic totally extraperitoneal (TEP) inguinal hernia repair: a single surgeon's experience. ANZ J Surg 2011;81:154158.

16) Berney CR, Descallar J. Review of 1000 fibrin glue mesh fixation during endoscopic totally extraperitoneal (TEP) inguinal hernia repair. Surg Endosc 2016;30:4544-4552.

17) Pascual G, Sotomayor S, Rodriguez M, et al. Cytotoxicity of $\mathrm{Cy}-$ anoacrylate-Based Tissue Adhesives and Short-Term Preclinical In Vivo Biocompatibility in Abdominal Hernia Repair. PLoS One 2016;11:e0157920.

18) Helbling C, Schlumpf R. Sutureless Lichtenstein: first results of a prospective randomised clinical trial. Hernia 2003;7:80-84.

19) Kukleta JF, Freytag C, Weber M. Efficiency and safety of mesh fixation in laparoscopic inguinal hernia repair using n-butyl cyanoacrylate: long-term biocompatibility in over 1,300 mesh fixations. Hernia 2012;16:153-162.

20) Subwongcharoen $S$, Ruksakul K. A randomized controlled trial of staple fixation versus N-butyl-2-cyanoacrylate fixation in laparoscopic inguinal hernia repair. J Med Assoc Thai 2013;96 Suppl 3: S8-13.

21) Jani K. Randomised controlled trial of $n$-butyl cyanoacrylate glue fixation versus suture fixation of mesh in laparoscopic totally extraperitoneal hernia repair. J Minim Access Surg 2016;12:118-123.

22) O’Reilly EA, Burke JP, O'Connell PR. A meta-analysis of surgical morbidity and recurrence after laparoscopic and open repair of primary unilateral inguinal hernia. Ann Surg 2012;255:846-853. 